\title{
Endovascular Thrombectomy in Patients with Intracranial Atherosclerosis: Where Are We?
}

\author{
Jong S. Kim \\ Stroke Center and Department of Neurology, Asan Medical Center, University of Ulsan College of Medicine, Seoul, Korea
}

Endovascular thrombectomy (EVT) has emerged as one of the most important treatment strategies for stroke patients with large vessel occlusion (LVO). However, previous studies rarely investigated LVOs associated with intracranial atherosclerotic disease (ICAD). Since large randomized trials were mainly conducted in the Western hemisphere, where ICAD is uncommon, the results may not be directly applicable to patients with ICAD.

South Korea is a typical Asian country where strokes associated with ICAD are highly prevalent. Nevertheless, the frequency of ICAD is not proportional to that of patients undergoing EVT. In the Asan Medical Center, one of the largest hospitals in Korea, patients with cardiogenic embolism (CE) accounted for 57\% of the patients who underwent EVT, whereas those with ICAD were only $24 \%$. 'This discrepancy is partly due to the fact that ICAD often produces lacune-like minor infarction associated with branch occlusion. Even when a complete main artery occlusion occurs, it often results in milder ischemic strokes than CE infarcts, probably because of the presence of well-developed collaterals.

Nevertheless, it should be realized that LVO associated with ICAD is relatively common, at least in Asian countries. In this regard, the comprehensive review by Lee et al., ${ }^{2}$ published in this issue, is quite timely. Based on this review and other recent studies, ${ }^{1}$ we have learned the following points:

1. Patients with ICAD have underlying stenotic vascular lesions and a highly thrombogenic environment; thus, they tend to re-occlude after thrombectomy. Therefore, several rescue treatment approaches are frequently needed, including intra-arterial infusion of antiplatelets and additional balloon angioplasty and/or stenting.

2. Perforator occlusion due to the snow-plowing effect might be one of the complications. However, this event may not be as serious as in cases of non-emergent angioplasty/stenting because the main vessel was already completely occluded in patients with LVO.

3. ICAD-related LVO tends to produce border-zone or scattered small infarcts rather than large, wedge-shaped territorial infarctions. Therefore, these patients often present with mild or fluctuating symptoms, depending on the collateral status, and arrive at the hospital later than those with CE. An article recently published in Journal of Stroke showed that patients with LVO associated with ICAD were generally treated later than CE patients; ICAD accounted for $19 \%$ of patients treated with EVT $\leq 24$ hours, but $46 \%$ of those treated with EVT $>24$ hours after onset. In this study, EVT in patients with ICAD in the late time window appears to be feasible and successful in selected patients. ${ }^{1}$

Nevertheless, the following questions should be resolved through further studies:

1. EVT may be performed beyond 24 hours from stroke onset in selected patients. Are there any objective criteria to identify patients who would benefit from EVT after 24 hours? If so, how long can the therapeutic time window be extended?

2. Antiplatelet therapy may be administered before EVT, considering the thrombogenic condition. However, possible bleeding complications should also be considered, especially in patients who receive intravenous tissue plasminogen activator (tPA). What is the best strategy? Would a fast-acting medication, such as ticagrelor, be more useful than clopidogrel?

3. Can we avoid using intravenous tPA in patients scheduled to undergo EVT?

4. Can we clearly differentiate between ICAD and non-ath- 
erosclerotic conditions, such as vasculitis, vasospasm, arterial dissection, and Moyamoya disease? Is EVT effective in these conditions as in ICAD?

In summary, EVT in ICAD patients has recently dramatically improved; however, we still have to go further to have answers for these important questions.

\section{References}

1. Lee D, Lee DH, Suh DC, Kim BJ, Kwon SU, Kwon HS, et al. Endovascular treatment in patients with cerebral artery occlusion of three different etiologies. J Stroke 2020;22:234-244.

2. Lee JS, Lee SJ, Hong JM, Alverne FJAM, Lima FO, Nogueira RG. Endovascular treatment of large vessel occlusion strokes due to intracranial atherosclerotic disease. J Stroke 2022;24:3-20.

Correspondence: Jong S. Kim

Department of Neurology, Asan Medical Center, University of Ulsan College of Medicine, 88 Olympic-ro 43-gil, Songpa-gu, Seoul 05505, Korea

Tel: +82-2-3010-3442

Fax: +82-2-474-4691

E-mail: jongskim@amc.seoul.kr

https://orcid.org/0000-0002-3999-4992

Received: January 5, 2022

Revised: January 6, 2022

Accepted: January 6, 2022

The author has no financial conflicts of interest. 